\title{
Comment on Pediatr Blood Cancer. 2022;e29589. Standardizing the surgical management of benign ovarian tumors in children and adolescents: A best practice Delphi consensus statement.
}

\author{
Matthew Murray ${ }^{1}$, Nigel Hall ${ }^{2}$, Sara Stoneham ${ }^{3}$, Anthony Penn ${ }^{4}$, Mark Brougham ${ }^{5}$, and \\ James Nicholson ${ }^{6}$ \\ ${ }^{1}$ Addenbrooke's Hospital \\ ${ }^{2}$ Southampton Children's Hospital \\ ${ }^{3}$ University College London Hospitals NHS Foundation Trust \\ ${ }^{4}$ Royal Manchester Children's Hospital \\ ${ }^{5}$ Royal Hospital for Sick Children \\ ${ }^{6}$ Adeenbrookes Hospital
}

March 7, 2022

Comment on Pediatr Blood Cancer. 2022;e29589. Standardizing the surgical management of benign ovarian tumors in children and adolescents: A best practice Delphi consensus statement.

Matthew J. Murray ${ }^{1,2}$, Nigel J. Hall ${ }^{3}$, Sara Stoneham ${ }^{4}$, Anthony Penn ${ }^{5}$, Mark Brougham ${ }^{6}$, James C. Nicholson ${ }^{1,7}$; on behalf of the Children's Cancer and Leukaemia Group (CCLG) Germ Cell Tumour (GCT) Special Interest Group (SIG).

${ }^{1}$ Department of Paediatric Haematology and Oncology, Cambridge University Hospitals NHS Foundation Trust, Cambridge, CB2 0QQ, UK

${ }^{2}$ Department of Pathology, University of Cambridge, Tennis Court Road, Cambridge, CB2 1QP, UK

${ }^{3}$ University Surgery Unit, Faculty of Medicine, University of Southampton, Southampton SO16 6YD, UK

${ }^{4}$ Department of Paediatrics and Child Health, University College Hospital London NHS Foundation Trust, London, UK

${ }^{5}$ Department of Paediatric Oncology, Royal Manchester Children's Hospital, Oxford Road, Manchester, M13 9WL, UK

${ }^{6}$ Department of Paediatric Oncology, Royal Hospital for Children \& Young People, 50 Little France Crescent, Edinburgh bio Quarter, Edinburgh, EH16 4TJ, UK

7 Department of Paediatrics, University of Cambridge, Cambridge University Hospitals NHS Foundation Trust, Cambridge, CB2 0QQ, UK

Conflict of interest statement : the authors declare no conflicts of interest.

We read with interest the article from Braungart and colleagues, discussing management of young females with benign ovarian tumors [1]. This is important and timely, and we agree with the need for clarity in management of this under-investigated group. As the authors note, published evidence to support decisions is limited, so in certain areas, guidance may have to rely on expert opinion. The authors emphasize the 
importance of multidisciplinary team (MDT) discussion to optimise management and risk-stratification [1]. They highlight the importance and safety of ovarian-sparing-surgery in benign tumors, to preserve fertility and avoid long-term effects including premature ovarian failure [1].

However, important aspects of their methodology are unclear. The denominator (number of experts approached to be involved) and response rate is not stated. The standard thresholds for accepting Delphi statements is $>70 \%$ votes in support from $>60 \%$ of experts in each voting round [2], as used in Delphi studies undertaken by the authors here [3,4]. Furthermore, there is no indication of level of support for statement(s) not reaching the $70 \%$ threshold. Moreover, they list specialties involved without defining numbers in each group, preventing assessment of the work's objectivity and relative representation. Frequent criticisms of Delphi method work are poor questionnaire/statement design, compounded by inadequate definition/ selection of experts [5]. For example, 'the inclusion of other clinicians... may be appropriate to provide an alternative clinical view, particularly when the study is expected to have an impact beyond a particular specialist field' [5]. Of particular note, the study states that a '.... Delphi panel was instigated that included... pediatric oncologists representing CCLG Germ Cell Tumour Group' [1]. Unfortunately, there was no pediatric oncologist involvement from the group in formulating the statements for discussion. Subsequently, only one was invited to participate in the Delphi itself, once the statements had been finalized. Furthermore, there was no patient/parent representation in the Delphi process, crucial to involve for a benign disease, with issues regarding surgical approach, fertility, and the burden of proposed ultrasound follow-up.

The surgical guidance is useful; however, a size cut-off for minimally invasive surgery is specified, without supportive evidence [1]. Another incongruity is for tumor marker follow-up (section 2.3, bullet 7) if they were elevated preoperatively; by definition this group should have had normal markers at diagnosis (section 2.1, first paragraph) [1]. In the follow-up section (2.3), bullet-points 3-5 are most contentious as no evidence is presented for dictating time-interval to scan, and a recent systematic review looking at this indication, which concluded, based on available evidence, that 'routine surveillance... should certainly be considered' and suggested annual ultrasound [6], was overlooked. In addition, no reference is provided here to existing guidelines [7]. The recommended two-year interval between ultrasounds [1] is therefore neither evidence-based nor considers the patient/parent opinion. Furthermore, there is no justification for gynecologic fertility referral following ovarian-sparing-surgery where subsequent ultrasound shows normal ovarian reserve bilaterally. Consequently, terms relating to specific aspects that 'should' include/be undertaken/performed are inappropriate. Suggesting that 'a reasonable strategy that could be considered might be as follows...' would be a better approach.

In summary, attempts to improve the management of young females with benign ovarian tumors are laudable. Some of the surgical recommendations made should help to streamline care. However, a representative group of key stakeholders were not included in the Delphi method and most of the follow-up recommendations cannot be justified based on use of a limited group of experts with limited supporting evidence. What is required is a wider collaborative approach, including GCT, surgical, gynecologic, radiology, and importantly patient groups, to answer these questions more robustly, preferably with international collaboration. This will also ensure appropriate clinician engagement, minimise inconsistencies in management and optimise outcomes for this patient group.

\section{References}

1. Braungart, S., et al., Standardizing the surgical management of benign ovarian tumors in children and adolescents: A best practice Delphi consensus statement. Pediatr Blood Cancer, 2022: p. e29589.

2. Walker, D.A., et al., A multi-disciplinary consensus statement concerning surgical approaches to lowgrade, high-grade astrocytomas and diffuse intrinsic pontine gliomas in childhood (CPN Paris 2011) using the Delphi method. Neuro Oncol, 2013. 15 (4): p. 462-8.

3. Murray, M.J., et al., Consensus on the management of intracranial germ-cell tumours. Lancet Oncol, 2015. 16 (9): p. e470-e477. 
4. Murray, M.J., et al., Delphi method to identify expert opinion to support children's cancer referral guidelines. Arch Dis Child, 2020.105 (3): p. 241-246.

5. Jones, J. and D. Hunter, Consensus methods for medical and health services research. BMJ, 1995. 311 (7001): p. 376-80.

6. Kiely, D., et al., Prevalence of metachronous contralateral mature ovarian teratoma: A systematic review. Pediatr Blood Cancer, 2021. 68 (11): p. e29237.

7. Brougham M, Nicholson J, Stoneham S, et al. Children's Cancer and Leukaemia Group; June 2018., Interim Guidelines for the Treatment of Extra CranialGerm Cell Tumours in Children and Adolescents. 2018. Accessed $15^{\text {th }}$ February 2022. 